\title{
繁 \\ Desempenho produtivo de vacas de corte cruzadas e zebuínas e seus bezerros
}

[Productive performance of crossbred and zebu cows and their calves]

\section{"Artigo Científico/Scientific Article"}

\author{
Emizael Menezes Almeida ${ }^{\text {** }}$, Marcelo Marcondes Godoy ${ }^{2 *}$, Alan Soares Machado ${ }^{2}$, \\ Douglas Dijkstra ${ }^{2}$, Reginaldo Martins Sousa ${ }^{2}$, Everton Oliveira Santos ${ }^{2}$, \\ Silvio Rosa Cardoso Neto ${ }^{2}$, Antonio Leandro Chaves Gurgel ${ }^{1}$
}

\footnotetext{
${ }^{1}$ Programa de Pós-Graduação em Ciência Animal, Universidade Federal de Mato Grosso do Sul, Campo Grande-MS, Brasil. ${ }^{2}$ Instituto Federal Goiano, Câmpus Ceres, Ceres-GO, Brasil.

*Autor para correspondência/Corresponding author: E-mail: emizaelmenezes@ gmail.com
}

\section{Resumo}

O objetivo do estudo foi avaliar o desempenho produtivo, produção de leite e os parâmetros da curva de lactação de vacas de corte e bezerros de três diferentes grupos genéticos no período pós-parto. Foram usadas 15 vacas paridas divididas em três grupos genéticos: cinco vacas $1 / 2$ Angus $1 / 2$ Nelore (F1), cinco 1/4Angus 1/4Nelore 1/2Tabapuã (F2) e cinco Nelore (NE). Avaliou-se a produção de leite, curva de lactação, peso corporal (PC), condição corporal (CC) e eficiência produtiva das vacas além do ganho de peso diário médio (GPDMB) e peso corporal no pós-parto e ao desmame (PCB) dos bezerros. As vacas F2 apresentaram maior PC comparado às $\mathrm{F} 1$ e NE que não diferiram entre si. $\mathrm{O}$ PCB do F2 e F1 não diferiram e, foram superiores aos NE dos 125 até 183 dias pós-parto. A CC das vacas F1 e F2 reduziu no período pós-parto, ao contrário das NE que mantiveram. Os bezerros mestiços tiveram melhor desempenho produtivo que o Nelore. A curva de lactação teve comportamento quadrático e parecido entre os grupos genéticos, assim como suas eficiências produtivas. As vacas F2 e F1 apresentaram maior produção de leite total e produção de leite diária em relação às vacas NE. Vacas mestiças, apesar das características produtivas superiores, tiveram eficiência produtiva parecida à raça Nelore, justificando o seu uso como matriz em sistemas de cruzamento com outras raças bovinas.

Palavras-chave: curva de lactação; mestiças; nelore; produção de leite.

\begin{abstract}
The aim of the study was to evaluate the productive performance, milk yield, and lactation curve parameters of beef cows and calves from three different genetic groups in the postpartum period. We used 15 calved cows divided into three genetic groups: Five 1/2Angus 1/2Nelore (F1), five 1/4Angus $1 / 4$ Nelore $1 / 2$ Tabapuã (F2), and five Nellore (NE) cows. We evaluated the milk yield, lactation curve, body weight, body condition (BC), and productive efficiency of cows beyond the average daily weight gain (AWGC), and body weight postpartum and at weaning (BWC) for the calves. The F2 cows had higher PC compared to F1 and NE, being that F1 and NE did not differ from each other. The F2 and F1 BWC did not differ from each other and these were both higher than NE from 125 to 183 days postpartum. The $\mathrm{BC}$ of the $\mathrm{F} 1$ and $\mathrm{F} 2$ cows reduced in the postpartum period, unlike the NE that remained unchanged. The crossbred calves had better performance than the Nellore. The lactation curve was quadratic and it had a similar behavior among genetic groups, as did their production efficiencies. The F2 and F1 cows showed higher total and daily milk yield compared with NE cows.
\end{abstract}


Crossbred cows, despite their superior production characteristics, have similar production efficiency to Nellore, justifying their use as a matrix in crossbreeding systems with other breeds.

Keywords: crossbreed; lactation curve; milk production; Nellore.

\section{Introdução}

O rebanho nacional de bovinos, no ano de 2016, contava com 218,23 milhões de cabeças, crescimento de $1,4 \%$ quando comparado ao ano anterior. A pecuária brasileira vem ocupando posição de destaque no cenário internacional, sendo consagrada como o segundo maior produtor mundial de carne bovina e o maior exportador (IBGE, 2016).

$\mathrm{O}$ aumento da produtividade na pecuária de corte de ciclo completo pode ser alcançado pelo uso de tecnologias e ferramentas que buscam a melhoria das condições para as vacas e sua prole ao longo do ano (Restle et al., 1999; Vieira et al., 2005). Uma ferramenta que vem ganhando força e cada vez mais utilizada pelos criadores brasileiros, e que vem gerando bons resultados é o cruzamento entre raças, com o objetivo de se usufruir do ganho de heterose, além da complementaridade das características (ABIEC, 2010).

A eficiência produtiva do rebanho de corte, na fase de cria, está associada ao desempenho reprodutivo, o genótipo da vaca, a habilidade materna da vaca, o genótipo do bezerro, o meio ambiente no qual vaca e bezerro vivem e a conversão da produção do leite em ganho de peso dos bezerros, um dos primeiros indicativos do potencial de crescimento (Ribeiro et al., 2001; Oliveira et al., 2007; Vaz et al., 2010).

A produção de leite da vaca de corte é de extrema importância, pois esse é utilizado com o objetivo de atender à exigência nutricional do bezerro principalmente durante os primeiros tempos de vida, o que reflete no seu desenvolvimento (Restle et al., 2005) e consequentemente no seu peso à desmama (Oliveira et al., 2007).

Objetivou-se, com este estudo, avaliar o desempenho produtivo das vacas de corte e seus bezerros, assim como os parâmetros de curva de lactação de vacas de corte de três diferentes grupos genéticos ao longo dos dias pós-parto.

\section{Material e Métodos}

O experimento foi realizado na fazenda Casa Grande, no município de Nova Glória - GO, no período de janeiro a junho de 2012. Foram utilizadas 15 vacas recém-paridas com idade média de 5 anos, peso corporal médio de $466 \mathrm{~kg}$. Os animais foram divididos em três grupos genéticos de cinco vacas, sendo: $1 / 2$ Angus $1 / 2$ Nelore (F1), 1/4Angus 1/4Nelore 1/2Tabapuã (F2) e Nelore (NE), e seus bezerros, filhos de touros Nelore, respectivamente. Os grupos de animais foram formados procurando uniformidade quanto à idade dos bezerros, peso e condição corporal das vacas.

As unidades experimentais (vacas com cria ao pé) foram manejadas em uma área de 27 ha, dividida em três piquetes de 9 ha. O sistema de pastejo adotado foi o rotacionado, com sete dias de ocupação e 14 dias de descanso. Os piquetes eram formados por Urochloa brizantha cv. Marandu, a lotação média durante todo o experimento foi de 0,8 UA/há, pois cinco vacas que não faziam parte do experimento foram manejadas juntamente com os animais do experimento. $\mathrm{O}$ sal mineral foi disponibilizado à vontade, para todos os animais.

Para avaliação da massa de forragem e valor nutritivo da pastagem, foram realizadas coletas de forragem a partir de simulação de pastejo e coleta total.

A coleta total foi realizada a cada 13 dias em 15 pontos diferentes de cada piquete, onde foi lançado um quadrado aleatoriamente com $0,25 \mathrm{~m}^{2}$ e foi realizado o corte da forragem rente ao solo. Posteriormente ao corte o material foi pesado e separado manualmente em lâminas foliares, colmo e material morto.

Estas frações foram secas em estufa de circulação forçada de ar a $60^{\circ} \mathrm{C}$ e pesadas. $\mathrm{O}$ componente lâmina foliar, após a secagem, foi triturado em moinho tipo Willey com peneira de 1 $\mathrm{mm}$. Os teores de matéria seca (MS) e proteína bruta $(\mathrm{PB})$ das lâminas foliares foram realizadas segundo Silva e Queiroz (2002). A fibra em detergente neutro (FDN) e fibra em detergente ácido (FDA) foram realizadas segundo Van Soest et al. (1991). Os nutrientes digestíveis totais (NDT) foram calculados segundo o método descrito por Capelle et al. (2001) em que: NDT $=83,79-$ $0,4171 *$ FDN. Os valores de massa seca total, 
massa seca de lâminas foliares, massa seca de lâminas foliares disponível para cada $100 \mathrm{~kg}$ de peso vivo, relação folha:colmo e valor nutritivo de lâminas foliares estão apresentados na Tabela 1 .

Tabela 1. Valores médios de massa seca total (MST), massa seca de lâminas foliares (MSF), massa seca de lâminas foliares disponível para cada $100 \mathrm{~kg}$ de peso vivo (MSF/100 kg PV), relação folha:colmo (RFC) e valor nutritivo de lâminas foliares da forragem Urochloa brizantha cv. Marandu durante os períodos experimentais.

\begin{tabular}{|c|c|c|c|c|c|}
\hline \multirow{2}{*}{ Variável } & \multicolumn{5}{|c|}{ Piquete } \\
\hline & \multicolumn{2}{|c|}{ Piquete 1} & \multicolumn{2}{|c|}{ Piquete 2} & Piquete 3 \\
\hline $\operatorname{MST}\left(\mathrm{kg} \mathrm{ha}^{-1}\right.$ de MS) & \multicolumn{2}{|c|}{3.746} & \multicolumn{2}{|c|}{3.746} & 1.936 \\
\hline $\operatorname{MSF}\left(\mathrm{kg} \mathrm{ha}^{-1}\right.$ de MS) & \multicolumn{2}{|c|}{636,8} & \multicolumn{2}{|c|}{1.158} & 346,6 \\
\hline $\mathrm{MSF} / 100 \mathrm{~kg} \mathrm{PV}$ & \multicolumn{2}{|c|}{38,7} & \multicolumn{2}{|c|}{32,4} & 20,0 \\
\hline RFC & \multicolumn{2}{|c|}{0,75} & \multicolumn{2}{|c|}{1,12} & 1,14 \\
\hline Piquete & MS & NDT & PB & FDN & FDA \\
\hline Piquete 1 & 35,26 & 52 & 5,72 & 76,2 & 38,18 \\
\hline Piquete 2 & 25,03 & 54,59 & 6,06 & 70 & 35,94 \\
\hline Piquete 3 & 29,02 & 57,2 & 6,86 & 63,75 & 32,05 \\
\hline Pastejo simulado & 34,7 & 52,54 & 7,14 & 74,9 & 37,7 \\
\hline
\end{tabular}

MS: matéria seca, NDT: nutrientes digestíveis totais, PB: proteína bruta, FDN: fibra em detergente neutro, FDA: fibra em detergente ácido e RFC: relação folha:colmo.

A pesagem e a avaliação da condição corporal (CC) dos animais foram realizadas individualmente aos 35 dias após o parto e a cada 30 dias até o desmame (183 dias de idade). Em cada pesagem, as vacas foram avaliadas quanto à condição corporal levando em consideração escala de 1 (muito magra) a 9 (muito gorda). O ganho de peso médio diário (GMD, $\left.\mathrm{kg} \mathrm{dia}^{-1}\right)$ dos bezerros foi calculado pela diferença de peso dos animais, dividido pelo número de dias entre as pesagens.

Para aferir os parâmetros relacionados à lactação foi utilizado o método direto (ordenha manual). Para isso, as vacas eram separadas dos bezerros um dia antes da ordenha e soltas em piquete anexo. Por volta das $18 \mathrm{~h}$, eram recolhidas até o centro de manejo, permanecendo com o bezerro por 30 minutos, tempo suficiente para esgotar o úbere. Após, os bezerros eram separados das vacas e ficavam contidos no centro de manejo e as vacas eram soltas novamente no piquete anexo. No dia seguinte, às $6 \mathrm{~h}$, as vacas foram contidas em tronco de contenção e amarrada apenas sua perna direita traseira. Após a aplicação intravenosa de 2 $\mathrm{ml}$ de ocitocina na veia mamária, os tetos, quando necessário, foram higienizados com água e iniciada a ordenha dos dois tetos do lado direito do úbere. A produção obtida era multiplicada por dois, para se ter a produção do úbere, sendo também corrigida para 24 horas por intermédio da equação conforme Restle et al. (2003):
Produção leite $(\mathrm{L} /$ dia $)=\frac{\left.(\text { (produção dos dois quartos do úbere })^{*} 2\right)}{\text { Tempo (minutos) entre a última mamada e a ordenha }}$

A eficiência produtiva de bezerros $(\mathrm{kg} / 100$ $\mathrm{kg}$ de peso corporal das vacas) ao desmame foi obtido pelo cociente entre o peso corporal do bezerro e da vaca aos 183 dias após o parto, multiplicado por 100 .

As produções de leite mensuradas foram utilizadas para descrever os parâmetros da curva de lactação, conforme o NRC (2000) onde:

$\mathrm{k}=1 / \mathrm{T}$, sendo: $\mathrm{k}=$ taxa intermediária constante e $\mathrm{T}=$ semana onde ocorre o pico de produção.

$\mathrm{a}=1 /($ PLP $\times \mathrm{k} \times 2,72)$, sendo: $\mathrm{a}=$ taxa intermediária constante e PLP = produção de leite no pico de produção (kg/dia).

A produção de leite em cada período de 30 dias foi definida pela equação desenvolvida por Alencar et al. (1996): PLn = [(Ln + Ln-1) / 2] x 30, onde PLn é a produção de leite do período de 30 dias; Ln, a produção de leite estimada do dia; e n, correspondente ao dia de avaliação, $(35,65, \ldots, 183)$. A produção de leite total (PLT) de cada vaca foi estimada somando-se todas as PLn (PLT $=\Sigma$ PLn).

A persistência de lactação (L) foi definida como o decréscimo linear diário na produção de leite entre as épocas do pico de lactação $(\mathrm{P})$ e da desmama (Jenkins et al., 2000):

$\mathrm{L}=(\mathrm{P}-$ produção no dia da desmama $) /$ número de dias entre o pico e o final da lactação. 
$\mathrm{O}$ delineamento experimental foi inteiramente casualizado em esquema fatorial $3 \mathrm{x}$ 6 , sendo três parcelas (grupo genético da vaca), seis subparcelas (dias pós-parto). Foram analisadas as características de desempenho produtivo (peso corporal, ganho de peso corporal, ganho médio diário dos bezerros e, para as vacas, peso corporal e escore de condição corporal).

Os parâmetros de curva de lactação "a" e "k", semana de ocorrência do pico de lactação, produção de leite no pico de lactação, persistência de lactação, $\mathrm{kg}$ de bezerro/100 $\mathrm{kg}$ de vaca, produção de leite total e produção diária de leite, foram estimados para cada vaca e considerada como dependentes, sendo submetida à análise de variância, de acordo com o modelo que incluiu os efeitos de grupo genético, sexo do bezerro e interação sexo $\times$ grupo genético. Análise estatística e as médias das características foram comparadas pelo teste Tukey ao nível de 5\%.

\section{Resultados e Discussão}

\section{Desempenho produtivo}

Não houve interação entre os três grupos genéticos das vacas e os dias em lactação, também não houve diferença para o fator dias em lactação, para a variável peso corporal (PC) $(\mathrm{P}>0,05)$. Porém, houve diferença entre os três grupos genéticos de vacas $(\mathrm{P}<0,05)$, para o peso corporal (Tabela 2).

Tabela 2. Médias do peso corporal $(\mathrm{kg})$ de vacas de diferentes grupos genéticos nos dias em lactação.

\begin{tabular}{|c|c|c|c|c|c|c|c|}
\hline \multirow{2}{*}{ Grupo genético } & \multicolumn{6}{|c|}{ Dias em lactação } & \multirow{2}{*}{ Média } \\
\hline & 35 & 65 & 95 & 125 & 155 & 183 & \\
\hline Nelore & 419,8 & 437,0 & 434,4 & 448,0 & 447,8 & 384,2 & $438,20^{\mathrm{A}}$ \\
\hline $1 / 2$ Angus $1 / 2$ Nelore & 440,6 & 466,2 & 480,0 & 476,8 & 478,2 & 442,2 & $454,33^{\mathrm{A}}$ \\
\hline $1 / 4$ Angus $1 / 4$ Nelore $1 / 2$ Tabapuã & 474,4 & 494,8 & 510,6 & 546,8 & 507,2 & 499,6 & $505,53^{\mathrm{B}}$ \\
\hline
\end{tabular}

Médias seguidas de letras maiúsculas iguais na mesma coluna não diferem entre si, pelo teste Tukey ao nível de 5\%.

No presente estudo verificou-se que vacas NE apresentaram peso corporal similar às mestiças F1. Já as vacas $\mathrm{F} 2$ foram $51,2 \mathrm{~kg}$ mais pesadas que as vacas F1 e 67,33 kg que as vacas NE. Segundo Restle et al. (2003), vacas Nelores apresentam menor porte em relação às vacas mestiças. Assim, esses animais têm maiores requerimentos nutricionais, principalmente de mantença, em relação a animais de estrutura corporal mediana. Esse fator deve ser considerado em condições não favoráveis, pois impede os animais de expressar seu potencial genético.

Durante o experimento os animais não apresentaram perda de peso corporal $(\mathrm{P}>0,05)$.
Contudo, outros autores relataram perda de peso corporal em vacas de corte com cria ao pé no estágio inicial de lactação entre 42 a 90 dias. Neste período ocorre a maior produção de leite, em razão disso, as vacas têm maiores exigências nutricionais e estão sujeitas a perdas de peso (NRC, 1996; Restle et al., 2001; Cerdótes et al., 2004).

Quanto à condição corporal das vacas não foi verificada interação entre os três grupos genéticos vs. dias em lactação, também não houve diferença para o fator grupo genético da vaca para a variável condição corporal (CC) $(\mathrm{P}>0,05)$. Porém, houve diferença entre os dias em lactação $(\mathrm{P}<0,05)$, para a condição corporal (Tabela 3).

Tabela 3. Médias de condição corporal (CC) de vacas de diferentes grupos genéticos nos dias em lactação.

\begin{tabular}{lcccccccc}
\hline \multirow{2}{*}{ Grupo genético } & \multicolumn{7}{c}{ Dias em lactação } & Média \\
\cline { 2 - 6 } & $\mathbf{3 5}$ & $\mathbf{6 5}$ & $\mathbf{9 5}$ & $\mathbf{1 2 5}$ & $\mathbf{1 5 5}$ & $\mathbf{1 8 3}$ & \\
\hline Nelore & $3,5^{\mathrm{a}}$ & $4,0^{\mathrm{a}}$ & $3,9^{\mathrm{a}}$ & $4,0^{\mathrm{a}}$ & $3,8^{\mathrm{a}}$ & $2,9^{\mathrm{a}}$ & $3,82^{\mathrm{A}}$ \\
1/2Angus 1/2Nelore & $4,1^{\mathrm{ab}}$ & $4,1^{\mathrm{ab}}$ & $4,5^{\mathrm{a}}$ & $4,0^{\mathrm{ab}}$ & $3,8^{\mathrm{ab}}$ & $3,7^{\mathrm{b}}$ & $3,90^{\mathrm{A}}$ \\
1/4Angus 1/4Nelore 1/2Tabapuã & $4,4^{\mathrm{a}}$ & $4,4^{\mathrm{a}}$ & $4,5^{\mathrm{a}}$ & $4,2^{\mathrm{a}}$ & $3,9^{\mathrm{a}}$ & $3,8^{\mathrm{a}}$ & $4,20^{\mathrm{A}}$ \\
\hline
\end{tabular}

Médias seguidas de letras minúsculas iguais na mesma linha não diferem entre si. Médias seguidas de letras maiúsculas iguais na mesma coluna não diferem entre si, pelo teste Tukey ao nível de $5 \%$.

Neste estudo não foi observada perda significativa $(\mathrm{P}>0,05)$ de CC. Para as vacas F1 foi observado maior CC aos 95 dias pós-parto quando comparado com 183 dias pós-parto. 
Na Tabela 4, estão os dados de peso corporal médio dos bezerros nos diferentes dias pós-parto. Não houve interação significativa $(\mathrm{P}>0,05)$ entre $\mathrm{o}$ grupo genético e dias pós-parto, sendo que a evolução do peso corporal dos bezerros ao longo dos dias pós-parto foi linear para todos os tratamentos, porém os bezerros F1 e F2 apresentaram maiores peso corporal a desmama em comparação aos NE.

Tabela 4. Médias do peso corporal dos bezerros (kg), filhos de vacas de diferentes grupos genéticos no pós-parto.

\begin{tabular}{lcccccc}
\hline \multirow{2}{*}{ Grupo genético } & \multicolumn{5}{c}{ Dias pós-parto } \\
\cline { 2 - 7 } & $\mathbf{3 5}$ & $\mathbf{6 5}$ & $\mathbf{9 5}$ & $\mathbf{1 2 5}$ & $\mathbf{1 5 5}$ & $\mathbf{1 8 3}$ \\
\hline Nelore & $56,4^{\mathrm{Ae}}$ & $76,3^{\mathrm{Bde}}$ & $96,3^{\mathrm{Bcd}}$ & $114,8^{\mathrm{Bbc}}$ & $130,2^{\mathrm{Bb}}$ & $157,1^{\mathrm{Ba}}$ \\
1/2Angus - 1/2Nelore & $62,5^{\mathrm{Ae}}$ & $86,2^{\mathrm{ABd}}$ & $112,9^{\mathrm{Abc}}$ & $137,2^{\mathrm{Ab}}$ & $155,9^{\mathrm{Ab}}$ & $182,6^{\mathrm{Aa}}$ \\
1/4Angus 1/4Nelore 1/2Tabapuã & $72,1^{\mathrm{Ae}}$ & $97,7^{\mathrm{Ad}}$ & $125,3^{\mathrm{Ac}}$ & $146,1^{\mathrm{Ac}}$ & $168,2^{\mathrm{Ab}}$ & $199,6^{\mathrm{Aa}}$ \\
\hline
\end{tabular}

Médias seguidas de letras minúsculas iguais na mesma linha não diferem entre si. Médias seguidas de letras maiúsculas iguais na mesma coluna não diferem entre si, pelo teste Tukey ao nível de 5\%.

As médias do peso corporal dos bezerros nos diferentes grupos genéticos foram iguais aos 35 dias. Aos 65 dias pós-parto os bezerros filhos de vacas F2 apresentaram os maiores valores de peso corporal quando comparado aos bezerros filhos de vacas $\mathrm{NE}(\mathrm{P}<0,05)$, os bezerros filhos de vacas $\mathrm{F} 1$ apresentaram peso corporal intermediários aos bezerros filhos de vacas NE e F2. Dos 95 até 183 dias pós-parto os animais dos grupos genéticos F2 e F1 não diferiram entre si, porém tiveram maior peso corporal que os $\mathrm{NE}(\mathrm{P}<0,05)$.

Quanto aos grupos genéticos, os bezerros filhos de vacas F1 e F2 pesaram em média 25,5 e $42,5 \mathrm{~kg}$ a mais que os bezerros filhos de vacas NE, respectivamente $(\mathrm{P}<0,05)$. Esses resultados demonstraram que houve efeito aditivo de genes proporcionado por cruzamento entre raças com maior aptidão leiteira. Assim as maiores produções de leite das vacas F2 (8,12 L/dia) e F1 (7,0 L/dia) em relação às $\mathrm{NE}(5,52 \mathrm{~L} / \mathrm{dia})$, promoveu maiores pesos à desmama dos bezerros.

Ribeiro et al. (2001) observaram peso corporal de bezerros ao desmame para as raças puras Aberdeen Angus e Charolês e mestiços $1 / 2$ Aberdeen Angus x $1 / 2$ Nelore e $1 / 2$ Charolês x $1 / 2$ Nelore filhos de vacas primíparas $1 / 2$ Aberdeen Angus x $1 / 2$ Nelore e $1 / 2$ Charolês x $1 / 2$ Nelore da ordem de 123,7 a $147,5 \mathrm{~kg}$. Estes resultados são inferiores aos encontrados neste estudo. Porém estes autores relatam que vacas primíparas da raça Charolês produziram mais leite em relação às vacas da raça Aberdeen Angus e desmamaram bezerros mais pesados quando comparado à raça Aberdeen Angus.

Restle et al. (2004) em experimento com 217 dias de duração em pastagem cultiva (PC) e pastagem nativa $(\mathrm{PN})$ com vacas Charolês $(\mathrm{CH})$ e Nelore (NE) não encontraram diferença entre os grupos genéticos, houve diferença entre sistema de alimentação. $\mathrm{O}$ peso corporal de bezerros ao desmame para PC-CH 189,2 kg; PC-NE 180,2 kg; PN-CH 137,8 kg; PN-NE 123,2 kg. Esses autores atribuíram o maior peso corporal dos bezerros ao desmame, principalmente à maior produção de leite das vacas mantidas em PC que foi $21 \%$ superior em relação às mantidas em PN.

Buskirk et al. (1995) trabalharam com vacas Angus e Angus-Hereford primíparas até 214 dias pós-parto e observaram peso corporal médio de bezerros ao desmame de $192 \mathrm{~kg}$. Neste estudo foram encontrados resultados semelhantes para os grupos genéticos F2 e F1 (199,6 e 182,6 kg, respectivamente).

Já Oliveira et al. (2007) observaram peso corporal ao desmame (210 dias) em bezerros filhos de vacas Nelore com Touros Nelore de 138,8 kg. Estes resultados inferiores ao deste trabalho, possivelmente pela menor produção de leite dos animais quando comparado aos deste estudo. Já os bezerros mestiços filhos de vaca $1 / 2$ Simental $x$ $1 / 2$ Nelore e $1 / 2$ Limousin $\times 1 / 2$ Nelore com touros Simental e Brahman o peso corporal ao desmame foi 187,1 e $175,5 \mathrm{~kg}$, resultados semelhantes aos deste estudo.

Para Souza et al. (2000), ao avaliarem vacas Nelore, verificaram peso corporal de bezerros Nelore ao desmame (180 a 205 dias) entre 153,33 a $158,33 \mathrm{~kg}$. Valores semelhante aos encontrados neste experimento. Já Alencar et al. (1999) e Vieira et al. (2005) que encontram resultado superior ao deste estudo, verificaram peso corporal ao desmame para bezerros Nelore entre 170 a $195 \mathrm{~kg}$, com idade ao desmame variando entre 205 e 270 dias. Os maiores valores no peso corporal ao desmame encontrado por esses autores pode ser atribuído a maior idade ao desmame dos bezerros 
quando comparado à idade ao desmame desse trabalho.

Os dados de desempenho dos bezerros na fase de aleitamento são mostrados na Tabela 5. Não houve interação significativa $(\mathrm{P}>0,05)$ entre $\mathrm{O}$ grupo genético e dias pós-parto. $\mathrm{O}$ ganho médio diário (GMD) foi influenciado pelo grupo genético e dias pós-parto $(\mathrm{P}<0,05)$. Os bezerros filhos de vacas F2 e F1 apresentaram maior GMD ao desmame quando comparados aos bezerros filhos de vacas NE. O GMD dos bezerros ao longo dos dias pós-parto teve comportamento quadrático para os tratamentos F2 e F1 como pode ser verificado nas equações de regressão. Para os bezerros filhos de vacas NE os dias pós-parto não influenciaram significativamente no GMD.

Tabela 5. Médias para ganho médio diário $\left(\mathrm{kg} \mathrm{dia}^{-1}\right)$ dos bezerros, filhos de vacas de diferentes grupos genéticos no pós-parto.

\begin{tabular}{lcccccc}
\hline \multirow{2}{*}{ Grupo genético } & \multicolumn{3}{c}{ Dias pós-parto } & \multirow{2}{*}{ Média } \\
\cline { 2 - 5 } & $\mathbf{3 5 - 6 5}$ & $\mathbf{6 5 - 9 5}$ & $\mathbf{9 5 - 1 2 5}$ & $\mathbf{1 2 5 - 1 5 5}$ & $\mathbf{1 5 5 - 1 8 3}$ & \\
\hline Nelore & $0,498^{\mathrm{ab}}$ & $0,500^{\mathrm{ab}}$ & $0,463^{\mathrm{ab}}$ & $0,385^{\mathrm{b}}$ & $0,668^{\mathrm{a}}$ & $0,504^{\mathrm{B}}$ \\
1/2Angus - 1/2Nelore & $0,593^{\mathrm{a}}$ & $0,668^{\mathrm{a}}$ & $0,520^{\mathrm{a}}$ & $0,468^{\mathrm{a}}$ & $0,673^{\mathrm{a}}$ & $0,601^{\mathrm{A}}$ \\
1/4Angus 1/4Nelore 1/2Tabapuã & $0,640^{\mathrm{ab}}$ & $0,690^{\mathrm{ab}}$ & $0,609^{\mathrm{b}}$ & $0,553^{\mathrm{ab}}$ & $0,785^{\mathrm{a}}$ & $0,638^{\mathrm{A}}$ \\
\hline
\end{tabular}

Médias seguidas de letras minúsculas iguais na mesma linha não diferem entre si. Médias seguidas de letras maiúsculas iguais na mesma coluna não diferem entre si, pelo teste Tukey ao nível de $5 \%$.

Quanto ao grupo genético, os bezerros filhos de vacas $\mathrm{F} 1$ e $\mathrm{F} 2$ obtiveram os maiores valores de $\operatorname{GMD}(0,601$ e $0,638 \mathrm{~kg} / \mathrm{dia}$, respectivamente) que os bezerros filhos de vacas $\mathrm{NE} 0,504 \mathrm{~kg} / \mathrm{dia}$. Os bezerros filhos de vacas F1 e F2 apresentaram 0,097 a $0,134 \mathrm{~kg} / \mathrm{dia}$ de GMD a mais que os bezerros filhos de vacas NE, até os 183 dias pósparto $(\mathrm{P}<0,05)$. Entre 125 e 155 dias foi o período que os bezerros apresentaram menores valores de GMD. Este fato pode ser explicado pela vacinação dos animais contra febre aftosa, que causa uma resposta imune nos animais, podendo reduzir o consumo ou até mesmo reduzir a produção leiteira da mãe, ocasionando redução no desempenho.

Oliveira et al. (2007) observaram GMD para bezerros mestiços filhos de vacas $1 / 2$ Simental $\mathrm{x}$
$1 / 2$ Nelore e $1 / 2$ Limousin x $1 / 2$ Nelore da ordem de 0,724 e $0,687 \mathrm{~kg}$. Estes resultados são semelhantes aos encontrados neste estudo com F2 e F1.

\section{Parâmetros de curva de lactação}

Não houve efeito significativo $(\mathrm{P}>0,05)$ do grupo genético da vaca e do sexo do bezerro sobre os parâmetros de curva de lactação, parâmetro "a" (de escala), parâmetro "k" (de forma), "S" semana de ocorrência do pico de lactação, "P" produção de leite no pico de lactação, "L" persistência de lactação e $\mathrm{kg}$ de bezerro/100 kg de vaca. Para a "PL" produção de leite diária e "T183" produção de leite total em 183 dias, houve efeito do grupo genético da vaca $(\mathrm{P}<0,05)$ (Tabela 6$)$.

Tabela 6. Valores médios referentes aos parâmetros a e k e suas variáveis relacionadas à curva de lactação e respectivas médias para os diferentes grupos genéticos.

\begin{tabular}{lccccc}
\hline Parâmetros & Nelore & F1 & F2 & CV $(\boldsymbol{\%})$ & Valor $\boldsymbol{p}$ \\
\hline $\mathrm{A}$ & $0,5350^{\mathrm{a}}$ & $0,3096^{\mathrm{a}}$ & $0,4238^{\mathrm{a}}$ & 46,86 & 0,24 \\
$\mathrm{~K}$ & $0,0976^{\mathrm{a}}$ & $0,1564^{\mathrm{a}}$ & $0,0882^{\mathrm{a}}$ & 48,19 & 0,15 \\
$\mathrm{~S}, \mathrm{sem}$ & $13,31^{\mathrm{a}}$ & $8,65^{\mathrm{a}}$ & $13,82^{\mathrm{a}}$ & 37,12 & 0,17 \\
$\mathrm{P}, \mathrm{kg} / \mathrm{d}$ & $8,53^{\mathrm{a}}$ & $10,25^{\mathrm{a}}$ & $10,34^{\mathrm{a}}$ & 15,51 & 0,14 \\
$\mathrm{~L}, \mathrm{~g} / \mathrm{d}$ & $-50,0^{\mathrm{a}}$ & $-44,0^{\mathrm{a}}$ & $-74,0^{\mathrm{a}}$ & 36,6 & 0,08 \\
$\mathrm{PL}, \mathrm{kg} / \mathrm{d}$ & $5,52^{\mathrm{b}}$ & $7,0^{\mathrm{a}}$ & $8,12^{\mathrm{a}}$ & 31,04 & $<, 001$ \\
T183, kg & $1010,2^{\mathrm{b}}$ & $1281,0^{\mathrm{a}}$ & $1486,0^{\mathrm{a}}$ & 31,04 & $<, 001$ \\
Kg bez/100 kg de vaca & $35,64^{\mathrm{a}}$ & $37,61^{\mathrm{a}}$ & $40,27^{\mathrm{a}}$ & 10,39 & 0,23 \\
\hline
\end{tabular}

F1 = 1/2Angus 1/2Nelore, F2 = 1/4Angus 1/4Nelore 1/2Tabapuã, a = parâmetro de escala da curva; $\mathrm{k}=$ parâmetro de forma da curva; $\mathrm{S}=$ pico de lactação; $\mathrm{P}=$ produção de leite no pico de lactação; T183 = produção de leite total em 183 dias; PL = produção de leite diária; $\mathrm{L}=$ redução diária na produção de leite do pico de lactação à desmama, 183 dias; CV (\%) Coeficiente de Variação. Valor - $p$ : probabilidade de efeito significativo. Médias seguidas de letras minúsculas iguais na mesma linha não diferem entre si, pelo teste de Tukey ao nível de 5\%. 
Oliveira et al. (2007) comparam vacas das raças Nelore e mestiças $(1 / 2$ Simental x $1 / 2$ Nelore e $1 / 2$ Limousin $x 1 / 2$ Nelore) observaram que vacas Nelore tem maior valor para o parâmetro "a" (de escala) em relação às mestiças. As vacas Nelore tiveram melhor persistência de lactação e menor produção de leite no pico quando comparado com as mestiças, resultados que diferem aos deste trabalho. Quanto ao parâmetro "k" (de forma) da curva de lactação e quanto à época de ocorrência do pico de lactação as vacas Nelores não diferiram das mestiças. As produções médias diárias e total de leite foram obtidas com as vacas Nelore, são concordantes com os dados deste estudo.

Neste trabalho, as curvas de lactação obtidas para os três grupos genéticos (Figura 1) indicam que as vacas F2 e F1 tiveram produção de leite superior as vacas Nelore.

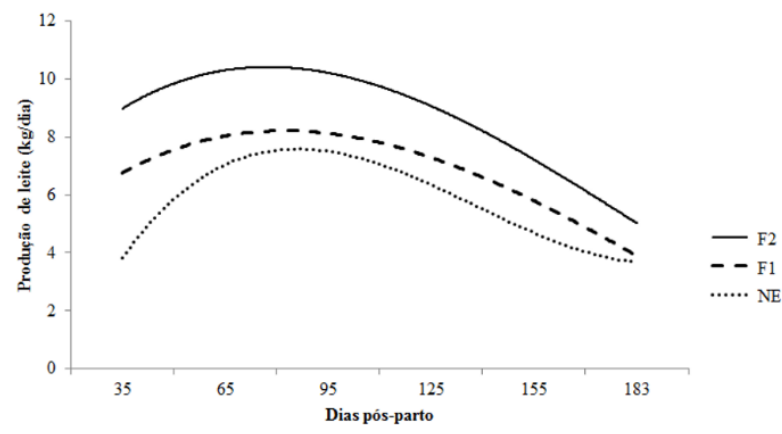

Figura 1. Curvas de lactação de vacas F2, F1 e NE.

O pico de lactação para as raças neste estudo foi 8,65 (F1), 13,31 (Nelore) e 13,82 (F2) semanas, esses dados corroboram com os resultados encontrados na literatura. O NRC (2000), estabeleceu que o pico de lactação de vacas de corte ocorre com 8,5 semanas de lactação. Oliveira et al. (2007) trabalharam com três raças, confinadas e duração de 210 dias e observaram a ocorrência do pico de lactação entre 10,8 e 14,3 semanas. Jenkins e Ferrell (1992), em um estudo com nove diferentes raças de vacas de corte (Angus, Braunvieh, Charolês, Gelbvieh, Hereford, Limousin, Red Poll, Pinzgauer e Simental), adotaram o modelo não linear para descrever a curva de lactação e constataram a ocorrência de pico de lactação entre 8,8 e 11,1 semanas pós-parto.

A produção de leite no pico foi similar aos resultados encontrados por Jenkins e Ferrell (1992) que relataram produção de leite entre 8,5 a 11,9 $\mathrm{kg} / \mathrm{dia}$ em nove diferentes raças taurinas de vacas de corte. Pimentel et al. (2006) obtiveram produção média de leite em vacas de corte multípara da raça Hereford 8,27 kg/dia. Já Oliveira et al. (2007) encontraram resultados semelhantes aos deste trabalho com vacas $1 / 2$ Simental $x 1 / 2$ Nelore que demonstrou a maior aptidão leiteira da raça Simental em relação às Limousin e Nelore.

As vacas F2 e F1 produziram mais leite $(\mathrm{P}<0,05)$ durante a lactação que as vacas da raça NE. Cerdótes et al. (2004) avaliaram a produção de leite em vacas mestiças $1 / 2$ Charolês $x 1 / 2$ Nelore ou $1 / 2$ Nelore $x 1 / 2$ Charolês, comparada às raças puras Nelore e Charolês a produção de leite nos cruzamentos foi superior quando comparados com as raças puras, e as mesmas não diferiram entre si. Ribeiro et al. (1991) obtiveram valores menores que os encontrados neste experimento para a produção de vacas Angus e Charolês em pastagem de azevém 3,08 a 3,50 kg/dia, respectivamente.

Para a produção de leite total as vacas mestiças tiveram maior produção $(\mathrm{P}<0,05) \mathrm{em}$ relação às Nelore. Valores menores foram encontrados por Oliveira et al. (2007) quando comparados aos resultados neste experimento para a produção de leite total em vacas Nelore (776,6 $\mathrm{kg}$ ) e semelhante às vacas $\mathrm{F} 1$ e F2 com vacas $1 / 2$ Simental x $1 \frac{1}{2}$ Nelore $(1422,5 \mathrm{~kg}$ ), sendo que esses autores estimaram a produção de leite total, em 210 dias de lactação, período esses de avaliação maior que o deste estudo. Ribeiro et al. (1991) em experimento com vacas Angus e Charolês obtiveram valores menores que os encontrados neste estudo para a produção de leite total. Já Jenkins e Ferrell (1992) observaram em vaca Angus produção de leite total de $1423 \mathrm{~kg}$. Estes resultados são semelhantes aos encontrados neste estudo com F1 e F2.

\section{Conclusão}

O cruzamento entre raças proporciona melhorias no desempenho produtivo dos animais. Vacas mestiças apresentam maior habilidade materna, expressa em maior produção de leite que refletem em maior ganho em peso corporal, ganho médio diário, maior peso à desmama dos bezerros em relação aos bezerros de vacas Nelore. A curva de lactação de vacas mestiças e puras apresentam comportamento semelhante.

\section{Conflito de Interesse}

Os autores declaram não existir conflito de interesse.

\section{Comitê de ética}

Esse estudo não possui protocolo gerado pela Comissão de Ética no Uso de Animais do 
Instituto Federal Goiano (CEUA-IFGoiano), pois de acordo com seu regimento interno, a referida Comissão passou a vigorar em dezembro de 2013 (Resolução $\mathrm{N}^{\circ}$ 050/2013 de 06 de dezembro de 2013) e o experimento foi realizado em 2012.

\section{Referências}

ABIEC. Associação Brasileira das Indústrias Exportadoras de Carnes. 2010. Rebanho Bovino Brasileiro. Disponível em: <http://www.abiec.com.br/3_rebanho.asp>. Acesso em: 17 fev. 2015.

Alencar, M.M.; Tullio, R.R.; Cruz, G.M.; Oliveira, M.C.S. Produção de leite da vaca e desenvolvimento do bezerro em gado de corte. Revista Brasileira de Zootecnia, 25(1): 92111, 1996.

Alencar, M.M.; Tullio, R.R.; Corrêa, L.A. Pesos e relações de peso de bezerros filhos de vacas Nelore e cruzadas Canchim x Nelore. Revista Brasileira de Zootecnia, 28(5): 968-973, 1999.

Buskirk, D.D.; Faulkner, D.B.; Ireland, F.A. Increased postweaning gain of beef heifers enhances fertility and milk production. Journal of Animal Science, 73(4): 937-946, 1995.

Capelle, E.R.; Valadares Filho, S.C.; Silva, J.F.C.; Cecon, P.R. Estimativas do valor energético a partir de características químicas e bromatológicas dos alimentos. Revista Brasileira de Zootecnia, 30(6): 1837-1856, 2001.

Cerdótes, L.; Restle, J.; Alves Filho, D.C.; Nörnberg, M.F.B.L.; Nörnberg, J.L.; Heck, I.; Silveira, M.F. Produção e composição do leite de vacas de corte de quatro grupos genéticos submetidas a dois manejos alimentares no período de lactação. Revista Brasileira de Zootecnia, 33(3): 610-622, 2004.

IBGE. Instituto Brasileiro de Geografia e Estatística. Produção da pecuária municipal. Rio de Janeiro, 44: 1-53, 2016.

Jenkins, T.G.; Ferrell, C.L. Lactation characteristics of nine breeds of cattle fed various quantities of dietary energy. Journal of Animal Science, 70: 1652-1660, 1992.

Jenkins, T.G.; Ferrell, C.L.; Roberts, A.J. Lactation and calf weight traits of mature crossbred cows fed varying daily levels of metabolizable energy. Journal of Animal Science, 78(1): 7$14,2000$.
NRC. National Research Council. Nutrient requirement of beef cattle. Washington: National Academy Press, 1996. 234p.

NRC. National Research Council. Nutrient requirements of beef cattle. $6^{\text {th }}$ ed. Washington, D.C.: National Academy Press, 2000. 242p.

Oliveira, V.C.; Fontes, C.A.A.; Siqueira, J.G.; Fernandes, A.M.; Sant'ana, N.F.; Chambela Neto, A. 2007. Produção de leite e desempenho dos bezerros de vacas Nelore e mestiças. Revista Brasileira de Zootecnia, 36(6): 2074-2081, 2007.

Pimentel, M.A.; Moraes, J.C.F.; Jaume, C.M.; Lemes, J.S.; Brauner, C.C. Características da lactação de vacas Hereford criadas em um sistema de produção extensivo na região da campanha do Rio Grande do Sul. Revista Brasileira de Zootecnia, 35(1): 159-168, 2006.

Restle, J. Desenvolvimento de bovinos de corte de diferentes grupos genéticos desmamados aos 3 ou 7 meses de idade. Revista Brasileira de Zootecnia, 28(5): 1023-1030, 1999.

Restle, J.; Vaz, R.Z.; Alves Filho, D.C.; Bernardes, R.A.L.C; Pascoal, L.L.; Senna, D.B.; Polli, V.A. Desempenho de vacas Charolês e Nelore desterneiradas aos três ou sete meses. Revista Brasileira de Zootecnia, 30(2): 499-507, 2001.

Restle, J.; Pacheco, P.S.; Moletta, J.L.; Brondani, I.L.; Certótes, L. Grupo genético e nível de nutricional pós-parto na produção e composição do leite de vacas de corte. Revista Brasileira de Zootecnia, 32(3): 585-597, 2003.

Restle, J.; Pacheco, P.S.; Pascoal, L.L.; Pádua, J.T.; Moletta, J.L.; Freitas, A.K.; Leite, D.T. Efeito da pastagem, da produção e da composição do leite no desempenho de bezerros de diferentes grupos genéticos. Revista Brasileira de Zootecnia, 33(3): 691-703, 2004.

Restle, J.; Pacheco, P.S.; Pádua, J.T.; Moletta, J.L.; Rocha, M.G.; Silva, J.H.S.; Freitas, A.K. Efeitos da taxa de ganho de peso pré-desmama de bezerras de corte e do nível nutricional pósparto, quando vacas, sobre a produção e composição do leite e o desempenho de bezerros. Revista Brasileira de Zootecnia, 34(1): 187-208, 2005.

Ribeiro, E.A.; Restle, J. Pires, C.C. Produção e composição do leite em vacas Charolês e Aberdeen Angus amamentando terneiros 
puros ou mestiços. Pesquisa Agropecuária Brasileira, 26(8): 1267-1273, 1991.

Ribeiro, E.L.A.; Restle, J.; Rocha, M.A.; Mizubuti, I.Y.; Silva, L.D.F. Eficiência produtiva em vacas primíparas das raças Aberdeen Angus e Charolês. Revista Brasileira de Zootecnia, 30(1): 125-132, 2001.

Silva, D.J.; Queiroz, A.C. Análises de alimentos (métodos químicos e biológicos). $3^{\mathrm{a}}$ ed. Viçosa, MG: Editora UFV, 2002. 235p.

Souza, J.C.; Ramos, A.A.; Silva, L.O.C.; Euclides Filho, K.; Alencar, M.M.; Wechsler, F.S.; Ferraz Filho, P.B. Fatores do ambiente sobre o peso ao desmame de bezerros da raça Nelore em regiões tropicais brasileiras. Ciência Rural, 30(5): 881-885, 2000.
Van Soest, P.J.; Robertson, J.D.; Lewis, B. A. Methods for dietary fiber, neutral detergent fiber, nonstarch polysaccharides in relation to animal nutrition. Journal of Dairy Science, 74(10): 3583- 3597, 1991.

Vaz, R.Z.; Lobato, J.F.P.; Restle, J. Productivity and efficiency of cow herds submitted to two weaning ages. Revista Brasileira de Zootecnia, 39(8): 1849-1856, 2010.

Vieira, A.; Lobato, J.F.P.; Correa, E.S.; Torres Junior, R.A.A.; Cezar, I.M. Produtividade e eficiência de vacas Nelore em pastagem de Brachiaria decumbens Stapf. nos Cerrados do Brasil Central. Revista Brasileira de Zootecnia, 34(4): 1357-1365, 2005. 